\title{
THE EFFECT OF SERUM CONCENTRATION ON THE SPHEROID FORMING ACTIVITY AND CELL GROWTH OF HUMAN MELANOCYTES ON CHITOSAN SURFACE
}

\author{
Sung-Jan Lin ${ }^{1,2,3,4}$ Wen-Chu HsiaO ${ }^{1}$, Chin-Jung Hsu ${ }^{2,4}$, JAU-Shiuh Chen ${ }^{2,4}$, \\ Shiou-Hwa JeE ${ }^{2,4}$, Tsen-Fang Tsai ${ }^{2,4}$, Hsin-Su Yu ${ }^{2,4}$, TAI-Horng Young ${ }^{1}$ \\ ${ }^{1}$ Institute of Biomedical Engineering, College of Medicine and Engineering, National Taiwan \\ University \\ ${ }^{2}$ Department of Dermatology, National Taiwan University Hospital, Taipei, Taiwan \\ ${ }^{3}$ Department of Dermatology, Yun-Lin Branch of National Taiwan University Hospital, Douliu, \\ Yunlin, Taiwan \\ ${ }^{4}$ Department of Dermatology, National Taiwan University College of Medicine, Taipei, Taiwan
}

\begin{abstract}
We have developed a chitosan-based melanocyte patch to improve the drawbacks encountered in autologous melanocyte transplantation for vitiligo treatment by use of melanocyte suspension. Melanocytes spontaneously grow into three dimensional spheroids on chitosan-coated surface and melanocyte spheroids show a better survival in growth factor and serum-deprived condition. Hence, preculturing melanocytes into spheroids on chitosan surface can provide melanocytes a survival advantage in stringent conditions. Up to date, the mechanism of the melanocyte spheroid formation is not clear. It has been shown that certain factors in serum are crucial for multicellular spheroid formation in cancer cell lines. In this work, we investigate the effect of serum concentration on the melanocyte spheroid formation. The trend for melanocyte spheroid formation is gradually decreased when serum concentration is serially increased from $0 \%$ to $20 \%$. Our result shows that melanocyte spheroid formation is not inhibited in the absence of serum and serum deprivation accelerates melanocyte spheroid formation. However, melanocyte growth is greatly reduced when serum is completely removed. Our results suggest that melanocyte spheroid formation on chitosan is not dependent on serum factors. However, the presence of serum contributes to the cell growth on chitosan-coated surface. This result is important in the future development of serum free culture system for spheroidal melanocyte patch.
\end{abstract}

Biomed Eng Appl Basis Comm, 2006(February); 18: 42-46.

Received: Oct. 25, 2005; Accepted: Nov. 28, 2006

Correspondence: Tai-Horng Young, Professor

Institute of Biomedical Engineering, National Taiwan

University, Taipei 100, Taiwan

E-mail: thyoung@ha.mc.ntu.edu.tw

\section{INTRODUCTION}

Vitiligo, characterized by depigmented patches on the skin, is an acquired disfiguring disease due to destruction of functional melanocytes in the epidermis [1-2]. Non-invasive treatment for vitiligo includes topical steroid, UV irradiation and lower energy irradiation [1-3]. These treatments are aimed at 
reactivating dormant melanocytes located in the hair follicle to repopulate the vitiliginous lesions [1,4-6]. However, these treatments may take months to more than a year before a favorable result is obtained. A surgical alternative employing autologous melanocyte transplantation has been proposed [2,7-10]. Autologous melanocytes are first expanded in culture condition. In the preparation of cells for transplantation, the cells are trypsinized into suspension. The cell suspension is then applied on the dermabraded depigmented lesions during transplantation. However, difficulty in handling the melanocyte suspension and variable successful repigmentation rates are often encountered [10].

To overcome drawbacks using melanocyte suspension, we have proposed the concept of using chitosan-based "melanocyte patch" for melanocyte transplantation [11-12]. The melanocyte patch is made by preculturing melanocytes on a chitosan membrane and the chitosan-based melanocyte patch can be used to cover the lesional sites with an upside-down orientation during transplantation. Chitosan supports the growth and phenotype expression of human melanocytes [11]. Further, in the presence fetal calf serum, human melanocytes form three dimensional multicellular spheroids on chitosan-coated surface when an appropriate seeding density is used [11]. Functionally, human melanocytes have a better survival in growth factor and serum deprived condition when they are precultured into multicellular spheroids on chitosan-coated surface [12]. Hence, chitosan-based spheroidal melanocyte patch may provide melanocytes a survival advantage in stringent conditions and it can theoretically improve the engraftment rates of melanocyte transplantation.

Up to date, the mechanism of melanocyte spheroid formation on chitosan membrane is unknown. It has been shown that certain serum signaling factors are crucial to multicellular spheroid formation in certain cancer cell lines [13-14]. It is notable that animal-derived serum in used in our culture system [11-12]. In terms of clinical application, the avoidance of animal serum in culture condition can prevent possible transfection of infectious disease from animal to human. However, if animal serum factors are critical in determining melancoyte spheroid forming activity, the use of animal derived serum may be mandatory to yield melanocyte spheroids in preparation of spheroidal melancoyte patch. In this work, we culture melanocytes on chitosan-coated wells in culture medium containing various concentrations of serum to shed light on the effect of serum-derived factors on the melanocyte spheroid formation and melanocyte growth.

\section{MATERIAL AND METHOD}

\subsection{Preparation of Culture Wells Coated With Chitosan}

A $15 \mathrm{mg} / \mathrm{ml}(\mathrm{W} / \mathrm{V})$ solution of chitosan (C-3646, Sigma, USA, Mn=810, $000 \mathrm{gm} / \mathrm{mole}$, degree of deacetylation $=85 \%$ ) was prepared by dissolving chitosan in $1 \mathrm{~m}$ acetic acid. For preparing chitosancoated wells, $0.5 \mathrm{ml}$ of chitosan solution was added into each well of 24-welled tissue culture polystyrene plates (Costar, USA). The solution was then allowed to dry at $50^{\circ} \mathrm{C}$ for 2 days to form a thin membrane. Each well was then neutralized by $0.1 \mathrm{~N} \mathrm{NaOH}$ aqueous solution for $15 \mathrm{~min}$ and washed thoroughly with double-distilled water. Before cell culture, the prepared chitosan-coated wells were sterilized in $70 \%$ alcohol overnight and rinsed extensively with phosphate buffered saline (PBS), followed by treatment under ultraviolet light overnight.

\subsection{Cell Culture, Serum Concentration and Spheroid Formation}

Human melanocytes were provided by cell bank of Department of Dermatology, National Taiwan University Hospital. The study protocol was approved by our Institutional Review Board. We conformed to the Helsinki Declaration with respect to human subjects in biomedical research. Human melanocytes are cultured using modified melanocyte medium (MMM) as we previously described [11]. Passage 1 or 2 melanocytes are detached by trypsinization before seeding. Cells were seeded evenly in the wells at the density of $25 \times 10^{3} / \mathrm{cm}^{2}$ in the following experiments. This seeding density was chosen because melanocytes could constantly grow into spheroids on chitosan surface in the presence of $20 \%$ fetal calf serum at this seeding density [11].

To investigate the behavior of melanocytes in various serum concentrations, melanocytes were cultured in MMM containing $0 \%, 1 \%, 2 \%, 5 \%, 10 \%$ and $20 \%$ fetal calf serum (Hyclone, USA) respectively and these experimental groups were designated as S0, $\mathrm{S} 1, \mathrm{~S} 2, \mathrm{~S} 5, \mathrm{~S} 10$ and S20 respectively in the following content. For morphological observation of melanocyte spheroid formation, the cells were photographed by digital camera coupled to a phase contrast microscopy after they were cultured for 2, 3, and 5 days respectively.

\subsection{Cell Proliferation Assays}

Quantitative comparison of cell growth was determined by MTT assays on day 1, 3, 5 respectively $[11,15]$, because counting of cells in the spheroids involves vigorous mechanical dissociation which 
damages the cells and results in a lower accuracy than automated quantization. The 300- $\mu 1$ MTT (Sigma, U.S.A.) solution ( $2 \mathrm{mg} / \mathrm{ml}$ in PBS) was added to each well. After $3 \mathrm{~h}$ incubation at $37^{\circ} \mathrm{C}$, the supernatant was discarded and dimethyl sulfoxide (Merck, Germany) of $200 \mu 1$ was added to dissolve the formazan crystals. The dissolvable solution was jogged homogenously for 15 min at room temperature by the shaker. The solution of each well was transferred to an eppendorf and was centrifuged at 1500 r.p.m. for $1 \mathrm{~min}$ to remove any possible melanocytes contaminated in the solution. The optical density of $100 \mu 1$ of the formazan solution was read on an ELISA plate reader (ELx800, BIOTEK) at $570 \mathrm{~nm}$.

\section{RESULT}

\subsection{The Effect of Serum Concentration on Melanocyte Spheroid Formation}

The behavior of melanocytes in chitosan-coated wells in different serum concentrations is shown in Figure 1, 2 and 3. On day 2, all groups showed initial aggregations on chitosan surface (Figure 1). The absence of serum did not inhibit the initial aggregation of melanocytes (Figure 1a). On day 3, more compact melanoycte spheroids were observed in groups with lower serum concentrations, especially S0 and S1 (Figure 2). The trend for formation of compact melancoyte spheroids seem to be enhanced by the decrease in serum concentration. This trend can be better appreciated during further culture period. As shown in Figure 3, more compact and larger spheroids could be revealed in groups with lower serum concentrations (Figure 3a, 3b and 3c) while increased serum concentrations seem to decrease the trend for spheroid formation (Figure 3d, 3e and 3f). Notably, in the absence of serum, the trend for melanocyte spheroid formation is enhanced to a greatest degree (Figure 1a, 2a, 3a).

\subsection{Cell Growth of Melanocytes in Various Serum Concentrations}

The result of MTT tests is shown in Figure 4. Analysis of the cell growth in various concentrations of serum reveals several interesting findings. In groups S2, S5, S10 and S20, a linear trend for cell growth was observed from day 1 to day 5 in culture. Judging from the results on day 5 , serum concentration increasing from $2 \%$ to $20 \%$ slightly enhances cell growth during the culture period for 5 days. In S1 group, though initial growth from day 1 to day 3 was revealed, the trend for cell growth was compromised from day 3 to day 5 . This result indicated that in serum concentration as low as $1 \%$, cell growth from day 3 to day 5 was not maintained to the level of cell growth from day 1 to day 3 .

In S0 group in which serum was completely deprived, cell growth further deteriorated. Though initial cell growth in S0 group was observed from day 1 to day 3, the degree of cell growth during this period in this group was lower in comparison with other groups with higher serum concentrations. In addition, no substantial cell growth from day 3 to day 5 was observed in S0 group, indicating that cell growth was almost arrested in this group during this period.

\section{DISCUSSION}

Cells are usually maintained as monolayered cells in vitro. However, cells can also grow into a three-

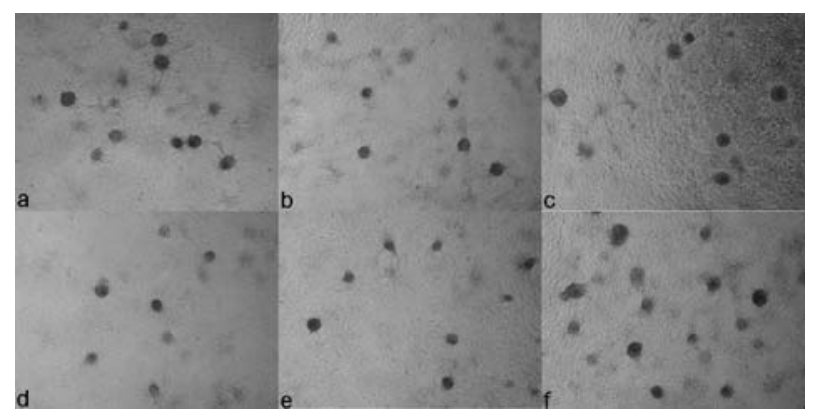

Fig 1. Cell morphology on day 2. (a) S0 (b) S1 (c) S2 (d) S5 (e) S10 (f) S20 (S0: serum concentration 0\%; S1: serum concentration 1\%; S2: serum concentration $2 \%$; $\mathrm{S} 5$ : serum concentration $5 \%$; S10: serum concentration 10\%; S20: serum concentration $20 \%$ ) (Phase contrast, $x 40$ )

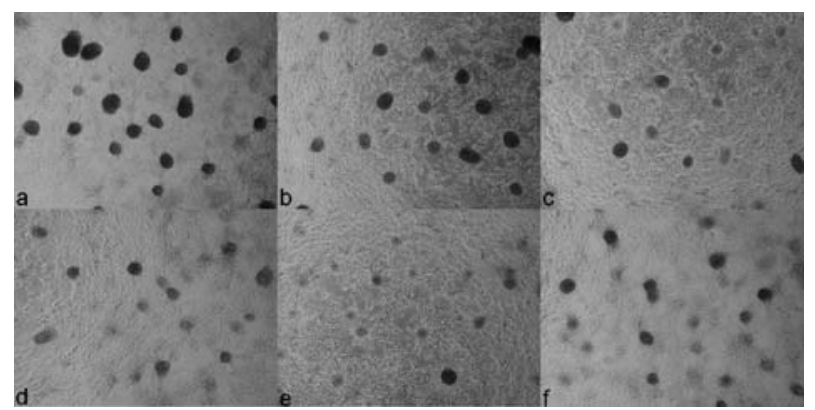

Fig 2. Cell morphology on day 3. (a) S0 (b) S1 (c) S2 (d) $\mathrm{S} 5$ (e) $\mathrm{S} 10$ (f) $\mathrm{S} 20$ (S0: serum concentration $0 \%$; S1: serum concentration 1\%; S2: serum concentration $2 \%$; S5: serum concentration 5\%; S10: serum concentration 10\%; S20: serum concentration $20 \%$ ) (Phase contrast, $x 40$ ) 
dimensional aggregate in the special environments, such as hepatocytes [16], neural stem cells [17], and certain cancer cell lines [13]. It has been shown that the function, proliferation and viability of cells are related to the morphology and intercellular organization of cells [17-19]. We have shown that, in comparison with monolayered melanocytes, melanocyte spheroids have a better survival in stringent conditions [12].

The behavior of human melanocytes on the biomaterial surface is characteristic. We have shown that at least two factors are important in determining melanocyte morphology: seeding density and substratum used. In the presence of $20 \%$ fetal calf

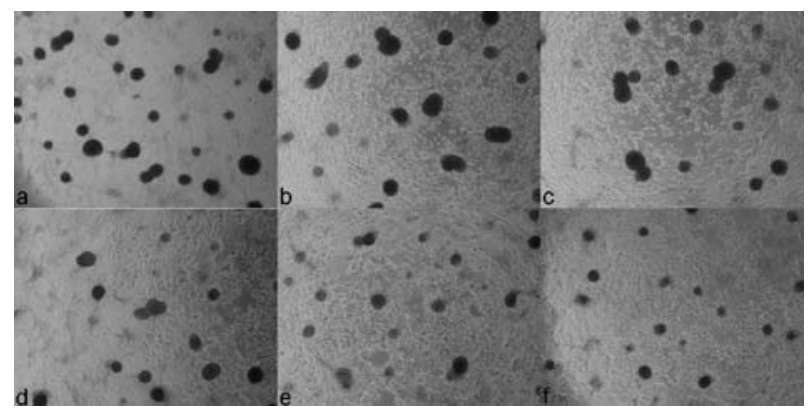

Fig 3. Cell morphology on day 5. (a) S0 (b) S1 (c) S2 (d) $\mathrm{S} 5$ (e) $\mathrm{S} 10$ (f) $\mathrm{S} 20$ (S0: serum concentration $0 \%$; S1: serum concentration 1\%; S2: serum concentration $2 \%$; $S 5$ : serum concentration $5 \%$; S10: serum concentration 10\%; S20: serum concentration $20 \%$ ) (Phase contrast, $x 40$ )

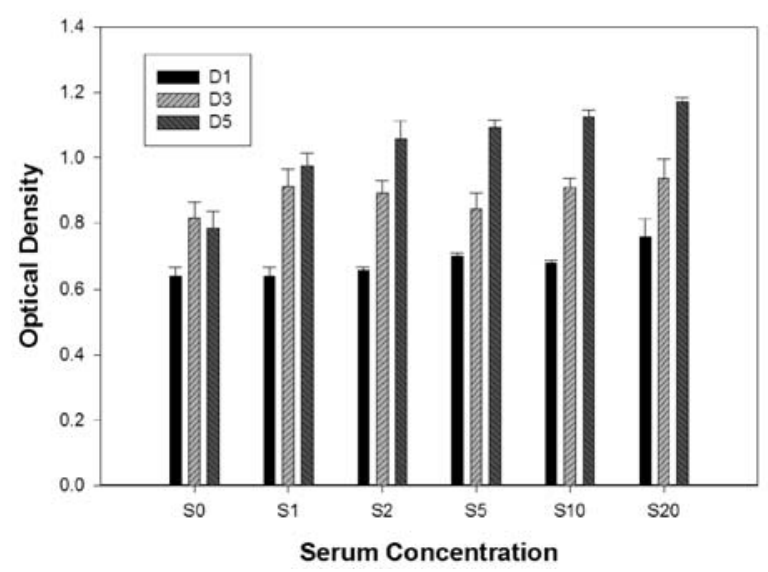

Fig 4. Cell growth in various serum concentrations from day 1 to day 5 . (S0: serum concentration $0 \%$; S1: serum concentration 1\%; S2: serum concentration $2 \%$; S5: serum concentration $5 \%$; S10: serum concentration 10\%; S20: serum concentration $20 \%$ ) serum when melanocytes are cultured on the chitosan surface, melanocytes can either be monolayered or spheroidal depending on the initial seeding density [11]. When initial seeding density exceeds a critical level, melanocytes spontaneously grow into spheroids within 3 days on chitosan surface [11]. In addition, in collagen-coated wells or tissue culture polystyrene wells, melanocytes can not maintain a spheroidal morphology [11-12].

How chitosan contributes to melanocyte spheroid formation was unknown in our previous studies. It has been shown in cancer cell lines that serum factors are crucial for spheroid formation [13-14]. It has also been shown that the behavior of cells on biomaterials can be controlled by serum-derived molecules adsorbed on the biomaterials, such as growth factors and adhesion molecules [20]. Hence, the special behavior of melanocytes on chitosan can also be affected by certain serum factors adsorbed onto the chitosan surface.

In this study, spheroid forming activity is enhanced when serum concentration is reduced. In the absence of serum, melanocytes grow into spheroidal morphology even faster. Hence, it is unlikely that factors derived from fetal calf serum play a major role in determining the morphology of melanocytes on chitosan surface. However, we still can not rule out that chitosan may contribute to spheroid formation by adsorbing factors secreted from melanocytes in high seeding densities.

On the other hand, serum concentration is important for melanocyte growth on chitosan surface. Judging from the results on day 5, there is a slightly increasing trend of cell growth when serum concentration is serially increased from $2 \%$ to $20 \%$. When serum concentration is lowered below the level of $2 \%$, cell growth on chitosan surface deteriorates. Though spheroid forming activity is highest in the absence of serum, cell growth is greatly reduced without serum. Hence, serum deprivation can be stringent for melanocyte growth in culture. Since serum deprivation helps melanocytes to aggregate, our result suggests that melanocyte may tend to adopt a spheroidal morphology in stringent conditions.

For clinical application, serum free culture can reduces possible transfection of infectious disease of animal origin by avoidance of animal derived serum. Our results confirm that serum factors in fetal calf serum are not crucial for spheroid forming activity of human melanocytes. Hence, in the future preparation of melanocyte spheroids for melanocyte transplantation, animal-derived serum can be minimized. However, other serum factors affecting melanocyte growth should be determined in further studies to keep a balance in maintaining spheroid forming activity and cell growth. 


\section{CONCLUSION}

The formation of melanocyte spheroid on chitosan-coated surface is not dependent on the presence of serum factors. On the contrary, spheroid forming activity is enhanced when serum is deprived. The tendency of melanocytes to aggregate into spheroids seems to decrease as the serum concentration is elevated. However, the presence of serum supports the sustained growth of melancoytes on chitosancoated surface. Our results are important in future development of serum free culture system for spheroidal melanocyte patch.

\section{REFERENCE}

1. Yu HS. Melanocyte destruction and repigmentation in vitiligo: a model for nerve cell damage and regrowth. J Biomed Sci 9: 564-573, 2002.

2. Njoo MD, Westerhof W, Bos JD, Bossuyt PMM. The development of guidelines for treatment of vitiligo. Arch Dermatol 135: 1514-1521, 1999.

3. Yu HS, Wu CS, Yu CL, Kao YH, Chiou MH. Helium-neon laser irradiation stimulates migration and proliferation in melanocytes and induces repigmentation in segmental-type vitiligo. J Invest Dermatol 120: 56-64, 2003.

4. Staricco RG. Activation of amelanotic melanocytes in the outer root sheath of the hair follicle following ultraviolet exposure. J Invest Dermatol 39: 163-164, 1962.

5. Ortonne JP, Schmitt D, Thivolet J. PUVA-induced repigmentation of vitiligo: Scanning electron microscopy of hair follicles. J Invest Dermatol 74: 40-42, 1980.

6. Cui J, Shen LY, Wang GC. Role of hair follicles in the repigmentation of vitiligo. J Invest Dermatol 97: 410-416, 1991.

7. Lontz W, Olsson MJ, Moellmann G, Lerner AB. Pigment cell transplantation for treatment of vitiligo: a progress report. J Am Acad Dermatol 30: 591-597, 1994.

8. ChenYF, Yang PY, Hung CM, Huang MH, Hu DN. Autotransplantation in segmental vitiligo by using cultured pure melanocytes: Analysis of 19 treated cases. Dermatol Sinica 17: 193-199, 1999.

9. Guerra L, Capurro S, Melchi F et al. Treatment of "stable" vitiligo by timedsurgery and transplantation of cultured epidermal autografts. Arch Dermatol 136: 1380-1389, 2000.

10. Yarr M. Vitiligo: the evolution of cultured epidermal autografts and other surgical treatment modalities. Arch Dermatol 137: 348-349, 2001.

11. Lin SJ, Jee SH, Shiao WC, Lee SJ, Young TH.
Formation of melanocyte spheroids on the chitosan-coated surface. Biomaterials 26: 14131422, 2005.

12. Lin SJ, Jee SH, Hsiao WC et al. Enhanced cell survival of melanocyte spheroids in serum starvation condition. Biomaterials (accepted).

13. Chun M. Plasmin induces the formation of multicellular spheroids of breast cancer cells. Cancer Lett 117: 51-56, 1997.

14. Chun MH. Serum signaling factors and spheroids. Critical Reviews in Oncology Hematology 36: 8998, 2000.

15. Mosmann T. Rapid colorimetric assay for cellular growth and survival: application of proliferation and cytotoxicity assays. J Immunol Methods 65: 55-63, 1983.

16. Koide N, Sakaguchi K, Koide Y et al. Formation of multicellular spheroids bcomposed of adult rat hepatocytes in dishes with positively charged surfaces and under other nonadherent environments. Exp Cell Res 186: 227-235, 1990.

17. Reynold BA, Weiss S. Clonal and population analysis demonstrate that an EGF-responsive mammalian embryonic CNS precursor is a stem cell. Dev Biol 175: 1-13, 1996.

18. Koide N, Shinji T, Tanabe T et al. Continued high albumin production by multicellular spheroids of adult rat hepatocytes formed in the presence of liver-derived proteoglycans. Biochem Bioph Res Co 161: 385-391, 1989.

19. Young TH, Huang JH, Huang SH, Hsu JP. The role of cell density in the survival of cultured cerebellar granule neurons. J Biomed Mater Res 52: 748-753, 2000.

20. Shelton RM, Rasmussen AC, Davies JE. Protein adsorption at the interface between charged polymer substrata and migrating osteoblasts. Biomaterials 9: 24-29, 1988. 